\title{
The structure of argumentation in health product messages
}

\author{
Douglas Walton* \\ CRRAR, University of Windsor, 2500 University Ave. W., Windsor, Ontario, Canada N9B $3 Y 1$ \\ (Received 14 October 2009; final version received 12 April 2010)

\begin{abstract}
This paper presents an analysis of argumentation in direct-to-consumer health product ads in Newsweek that brings out special features of the arguments used in the ads, including practical reasoning, chained arguments, enthymemes, and prolepsis. A way to help overcome deficiencies in techniques of tailored health communication in consumer health informatics is shown by using argumentation schemes, argument visualisation tools, and dialogue models to frame these persuasive communication messages. The evidence collected is shown to be useful to allow the health informatics communicator and the person to whom message was directed to interact in a dialogue format where arguments can be put forward by both parties in a way that responds to the previous arguments and questions of the other party.
\end{abstract}

Keywords: practical reasoning; argumentation schemes; Araucaria; persuasion dialogue; direct-to-consumer ads; consumer health informatics

In this paper, some illustrative examples of direct-to-consumer health product advertisements are analysed using argumentation methods, and some conclusions about the analyses are drawn. The three main methods applied are argumentation schemes, argument visualisation, and dialogue structures that represent the context of use of an argument. The ads studied include commercials for pharmaceuticals, as well as ads for other medications, including health foods, and treatments and devices that purport to have health benefits. It is shown that they use arguments of a kind that fit structures of kinds known in argumentation studies, especially one particular argumentation scheme. The findings are interesting for many reasons. They can be applied both to the crafting of the ads and to the enhancement of critical thinking skills for intelligent consumers. They are also applicable to recent efforts to use computational techniques to assist consumer health informatics to inform consumers and improve health care.

According to Kukafka (2005, p. 29), applying persuasive argumentation theories to consumer healthcare informatics for behavioural change has turned out to be a complex undertaking, because of the limitations of some of the tools used. The natural language generation systems used to provide explanation and advice to consumers have proved to have limitations and shortcomings. One problem is that they do not explore planning mechanisms that account for the generation of text consisting of multiple arguments. Another is that although they have attempted to improve the construction of persuasive argument through rhetorical structure theory, these attempts lack a theory of how persuasive arguments in health communication are put together in a coherent sequence. At the same time, the tailored approach to health communication, where the behavioural change strategy is intended to reach a specific person, although it is proving to be a successful method, clearly needs better argumentation tools that allow the health informatics communicator and the person to whom message was directed to interact in a dialogue format where arguments

*Email: dwalton@uwindsor.ca 
can be put forward by both parties in a way that responds to the previous arguments and questions of the other party.

This paper shows a way to help overcome these deficiencies by using argumentation tools, especially argumentation schemes, argument visualisation tools, and use of dialogue models to frame persuasive communication messages. Evidence collected and analysed in this paper, by examining some specimens of argumentation in a selection of some recent health product ads in Newsweek, is shown to be useful to help explore the planning mechanisms that account for the generation of text consisting of multiple arguments. The Newsweek ads are shown to be centrally based on the argumentation scheme for practical reasoning, chained with other kinds of arguments that can be represented by argumentation schemes. Practical reasoning is goal-directed reasoning, used where an agent concludes that a course of action should be carried out for the reason that it will contribute to the fulfilment of goals that the agent is committed to. Practical reasoning is used in artificial intelligence in helping human and artificial agents make intelligent deliberations on what to do in a given set of circumstances (Bratman, Israel, and Pollack 1988; Atkinson, Bench-Capon, and McBurney 2006). Use of practical reasoning of this sort as argumentation is commonly called the problem/solution strategy in advertising. It is shown how practical reasoning and its critical questions are key components in the dialogue structure of these communicative health messages.

\section{Enthymemes and argumentation schemes}

In this section, the reader is introduced to the argumentation tools that will be applied in the rest of the paper, including argumentation schemes, enthymemes and argument mapping tools. Argumentation schemes are standardised forms of reasoning representing stereotypical kinds of arguments (Walton, Reed and Macagno 2008), made up of standard premises and a standard conclusion representing forms of argument that are generally (but not always) defeasible. An example is: $E$ is an expert; $E$ says that statement $A$ is true; therefore $A$ can be tentatively accepted (subject to critical questioning and retraction in light of new evidence that may suggest otherwise). Enthymemes are arguments with implicit premises or conclusions (Walton and Reed 2005; Walton 2008). Argument mapping tools are devices used to visualise the premises and conclusions of arguments in a tree structure, and display a sequence of connected arguments chained together to support an ultimate conclusion. More than forty such tools are described by Scheuer, Loll, Pinkwart, and McLaren (2009). The one used in this paper, Araucaria (Reed and Rowe 2002), was the first to use argumentation schemes, and can be obtained from http://araucaria.computing.dundee.ac.uk/.

The most interesting schemes in current research are defeasible ones that represent plausible reasoning, rather than deductive or inductive reasoning. Each scheme has a special set of critical questions matching it. An argument is evaluated using the critical questions in relation to the scheme. The argument holds as plausible unless critically questioned or attacked by an opposing argument, or by the asking of a critical question.

An example of an enthymeme is: 'All men are mortal, therefore Socrates is mortal', where the implicit premise 'Socrates is a man' was not stated. But there is a problem (Ennis 1982; Burke 1985; Gough and Tindale 1985; Hitchcock 1985). If the analyst is allowed to fill in any proposition needed to make such an inference valid, he or she may be inserting assumptions into the text of discourse that the speaker did not mean to be part of his or her argument. There is even the danger of committing the straw man fallacy, the fallacy attributing an implicit premise or conclusion to an opponent's argument that exaggerates or distorts the argument in order to make it easier to refute (Scriven 1976, pp. 85-86). However, new methods of reconstructing enthymemes (Walton 2007a) have been shown to be useful in contending with this danger. 
Practical reasoning has for some time been recognised in AI as a common and highly significant form of a component of rational behaviour of the kind where actions are taken to further the goals of an agent, based on the agent's knowledge of its given set of circumstances (Bratman et al. 1988). There are various simpler and more complex versions of this scheme presented in the argumentation literature (Walton et al. 2008). In the version of the scheme presented below, the first-person pronoun 'I' represents a rational agent of the kind described by Wooldridge (2000), an entity that has goals, some (though possibly incomplete) knowledge of its circumstances, and the capability of acting to alter those circumstances and to perceive (some of) the consequences of so acting. This very simplest form of practical reasoning is called practical inference (Walton et al. 2008, p. 323).

Major premise: I have a goal $G$.

Minor premise: Carrying out this action $A$ is a means to realise $G$.

Conclusion: Therefore, I ought (practically speaking) to carry out this action $A$.

Below is the set of critical questions matching the scheme for practical inference (Walton et al. 2008, p. 323).

$\mathrm{CQ}_{1}$ : What other goals do I have that should be considered that might conflict with $G$ ?

$\mathrm{CQ}_{2}$ : What alternative actions to my bringing about $A$ that would also bring about $G$ should be considered?

$\mathrm{CQ}_{3}$ : Among bringing about $A$ and these alternative actions, which is arguably the most efficient?

$\mathrm{CQ}_{4}$ : What grounds are there for arguing that it is practically possible for me to bring about $A$ ?

$\mathrm{CQ}_{5}$ : What consequences of my bringing about $A$ should also be taken into account?

The last critical question, $\mathrm{CQ}_{5}$, is very often called the side effects question. It concerns potential negative consequences of a proposed course of actions. Just asking about consequences of a course of action being contemplated could be enough to cast an argument based on practical reasoning into doubt.

Critical question $\mathrm{CQ}_{5}$ is closely related to another scheme called argument from negative consequences, a form of counter-argument that cites the consequences of a proposed course of action as a reason against taking that course of action. Two argumentation schemes representing the two forms of argumentation from consequences have been put forward in (Walton 1996, p. 75). The following is the scheme for argument from positive consequences.

Premise: If $A$ is brought about, good consequences will plausibly occur.

Conclusion: A should be brought about.

The following is the scheme for argument from negative consequences.

Premise: If $A$ is brought about, bad consequences will plausibly occur.

Conclusion: $A$ should not be brought about.

According to (Walton 1996, pp. 76-77), three critical questions match the scheme.

CQ1: How strong is the probability or plausibility that these cited consequences will (may, might, must) occur?

CQ2: What evidence, if any, supported the claim that these consequences will (may, might, must) occur if $A$ is brought about?

CQ3: Are there consequences of the opposite value that ought to be taken into account? 
These forms of argument are defeasible, meaning that each of them is cast into doubt if there is a failure to answer any critical question adequately. Both schemes are built on the use of values in argumentation and are associated with schemes for argument from positive values and negative values.

The scheme for argument from negative value is from (Walton et al. 2008, p. 321).

Premise 1: Value $V$ is negative as judged by agent $A$ (judgment value)

Premise 2: The fact that value $V$ is negative affects the interpretation and therefore the evaluation of goal $G$ of agent $A$ (If value $V$ is $b a d$, it goes against commitment to goal $G)$.

Conclusion: $V$ is a reason for retracting commitment to goal $G$.

For value-based reasoning, see (Bench-Capon 2003; Bench-Capon and Atkinson 2009).

The simplest form of practical reasoning shown above is purely instrumental, in that it makes no reference to value-based argumentation. Another widely used variant of practical reasoning is called value-based practical reasoning (Atkinson et al. 2006). The version of this scheme below is from Walton et al. (2008, p. 324).

Premise 1: I have a goal $G$.

Premise 2: $G$ is supported by my set of values, $V$.

Premise 3: Bringing about $A$ is necessary (or sufficient) for me to bring about $G$.

Conclusion: Therefore, I should (practically ought to) bring about $A$.

Note that value-based practical reasoning can be classified as a hybrid scheme that combines argument from values with practical reasoning.

Another required component of the new method is the use of visualisation tools that can be used to help analyse an argument and that have argumentation schemes as part of the tool. Araucaria also supports argumentation schemes, and provides sets of schemes from which a user can select a scheme and apply it to a given argument. By applying this tool, schemes can be used as part of the technique for analysing an argument, and for displaying its structure in a visual form. Such an argument visualisation tool can be useful for many purposes. It can be used to summarise an argument, or as a device to help explain its inner workings.

\section{Frameworks of dialogue and fallacies}

An important tool needed for the analysis of the examples of the health product ads that follow is the specification of the context of dialogue. Each model of dialogue is defined by its initial situation, the participants' individual goals, and the collective goal of the dialogue as a whole (Walton and Krabbe 1995). Six basic types of dialogue previously recognised in the argumentation literature are persuasion dialogue, inquiry, negotiation dialogue, information-seeking dialogue, deliberation, and eristic dialogue. These dialogues are normative models, meaning that they do not necessarily correspond exactly to real instances of persuasion etc. that may occur in a real conversational exchange (Hamblin 1970). There is a formal model of persuasion dialogue (Prakken 2006).

A good initial approach to help see where we are going is to adopt the hypothesis that the argumentation structure of health product ads takes the basic form of a persuasion dialogue between the advertiser who designs the ad and publishes it, and the reader to whom the ad is directed. Each contributes to a part of the inference leading to the conclusion based on the argumentation scheme for practical reasoning. The advertiser targets a particular group of readers who are presumed to have some problem they need to solve, and hence are presumed to have a goal that can be determined. It is the reader who contributes this goal. The advertiser provides a means that the 


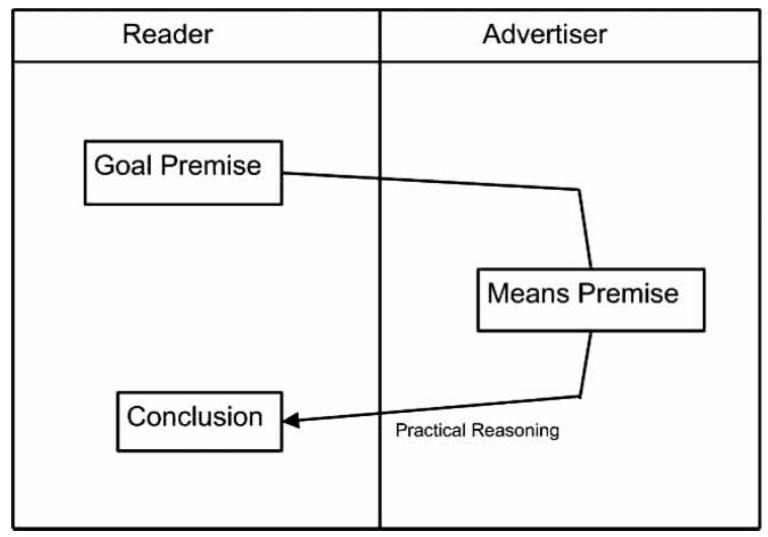

Figure 1. Persuasion dialogue in which two parties use practical reasoning.

reader can use to fulfil this goal, and presents this means to the reader. The reader is supposed to draw the conclusion that he can fulfil the goal, or at least take some steps towards its fulfilment, by drawing the conclusion that he should carry out the action recommended in the means premise. The structure of the argument as a whole is that of practical reasoning, as shown in Figure 1, which displays the two parties and indicates the role each has in the reasoning used to derive the conclusion. In this way, the two parties both take part in the reasoning process, even though they do not have a dialogue in the literal sense that they take turns speaking and replying. Still their argumentation interaction does have what might be called a dialogue structure in the sense of (Walton and Krabbe 1995), as shown in Figure 1. The advertiser, to make a successful argument that will persuade the reader to buy the product (or ask his doctor about it) needs to base it on what he takes to be the commitments of the reader, including the reader's presumed goals and values.

The structure of the argument shown in Figure 1 is very simple, but it represents a starting point in representing the basic dialogue structure of the ad.

Many health product ads contain pictures or other graphics as well as text. Birdsell and Groarke (1996) have studied visual ads that express arguments with premises and conclusions in very much the same way that arguments are put forward in written discourse. They admit that visual ads can be vague and ambiguous, and admit all sorts of difficulties about justifying an interpretation about what the argument is supposed to be. However, they point out that written messages in ads, or any natural language texts of discourse, are confronted with these problems too. They stress (Birdsell and Groarke 1996, p. 6) that context is very important with interpreting visual ads, including not only sequences of images, but also verbal context and implicit assumptions about what the readers of the ad can be expected to know or accept. Their findings suggest that the visual components of health product ads need to be taken into account.

Another dialectical aspect of a health product ad that needs to be taken into account is whether information-seeking dialogue needs to be embedded into the persuasion dialogue in order for the argumentation in the ad to be reasonable enough to meet standards for an acceptable argument of this sort. As an additional part of the practical reasoning strategy used in the ad, the advertiser must also provide information that can be used to answer the reader's critical questions. For example, in the case of some health products, the reader will be concerned about potential side effects of taking a medication. The advertiser needs to adopt the strategy of crafting the ad in such a way that critical questions, like those concerning negative consequences of carrying out the designated action, are responded to. Thus the advertiser's task is an empathic one of not only targeting a 
particular readership by formulating a goal, but also one of being aware of concerns that the reader will have that will provide reservations he or she would have about carrying out the action proposed in the conclusion.

There are controversies in evaluating the argumentation in the ads studied below on whether a failure to present information in an argument in an ad is improper, or even represents fallacious argumentation. An underlying issue with health product ads is whether the purpose of the ad is (or should be) (a) to persuade the reader to buy the product depicted in the ad, or (b) to give the reader information about the product in the ad. There is also the possibility that these aims are combined, and so we have to recognise the phenomenon called the dialectical shift in the argumentation literature (Walton and Krabbe 1995, pp. 100-116). In a common kind of example, there is a shift from deliberation dialogue to an information-seeking type of dialogue. For example, suppose there is a debate in a legislative assembly on whether to pass a bill to install a new dam, but before those in the assembly can vote on the issue, they need to find out many facts about the proposed dam and its projected costs. They will call in experts, like engineers and accountants. When this occurs there is a shift from the original deliberation dialogue to an information-seeking dialogue. This shift would be classified as an embedding in the system of (Walton and Krabbe 1995, p. 102), meaning that the goal of the deliberation is supported by the information-seeking dialogue. But some dialectical shifts are illicit, meaning that the advent of the second dialogue interferes with the progress of the first (Walton and Krabbe 1995, p. 107). Illicit shifts of this sort can be associated with fallacies, according to Walton and Krabbe (1995).

It is a commonplace of discussion of advertisements in visual media to claim that they use irrational appeals and faulty logic to sell products (Slade 2003). Logic textbooks have propounded this view for years by telling us that such ads tend to use emotional appeals that shortcut logical reasoning, and even commit logical fallacies. One of the most widely used logic textbooks (Copi and Cohen 2005, p. 128) tells us that commercial ads are designed by 'ballyhoo artists' who persistently use relentless illogical appeals to emotions of every kind. Recent studies of argumentation in pharmaceutical ads (Rubinelli 2005; Rubinelli, Nakamoto, and Schulz 2007, 2008) appear to confirm that these ads commit logical fallacies. Direct-to-consumer advertising (DTCA) of pharmaceutical products is illegal in all countries of the Western world except for the US and New Zealand (Calfee 2002, p. 176). However, it reaches all over the world through the Internet (Rubinelli et al. 2008, p. 49). Thus the question of whether they commit fallacies is significant and controversial.

Using methods of argumentation theory to analyse examples of ads for pharmaceutical products, Rubinelli (2005) concluded that some of the arguments in the ads can be said to have committed informal fallacies. She criticised an ad that failed to include information about side effects as having committed a fallacy of omission (Rubinelli 2005, p. 89). Rubinelli et al. (2007, p. 1213) also alleged fallacies of failing to present relevant information about side effects. Supporters of DTCA ads defend them, however, as a way of providing people with information about the benefits and risks of medication (Calfee 2002). Detractors (Lexchin and Mintzes 2002, p. 197) cite evidence that many ads surveyed did not contain the basic information a person would need to judge the usefulness of a treatment.

These allegations of committing fallacies stems from the perception that there is a failure to provide relevant information in DTCA ads. The assumption that the ads should provide information leaves them open to allegations of having committed fallacies of omission by not providing enough information (Rubinelli 2005, pp. 76-77). But how much is enough, and should the purpose of these ads be to present information, when we all know that a pharmaceutical company is not an unbiased source of information on the benefits or risks of the product it sells? Questions are raised here about the context of use of the ad for some communicative purpose in a dialogue setting. 


\section{Examples of fallacious arguments in ads}

Groarke (2009, section 10) analysed an example of a vodka ad in which a giant bottle of vodka is shown being poured onto a sleepy village, transforming it into a glittering city with skyscrapers. He analysed the message conveyed the picture (p. 2) as expressing the following argument:

Explicit premise: If you add vodka to your life, your sleepy life will be transformed into a life of cosmopolitan excitement;

Implicit premise: A life of cosmopolitan excitement is desirable;

Conclusion: $\quad$ You should add vodka to your life (i.e. purchase vodka).

Groarke (p. 2) concluded that this argument is fallacious, based on the reason that it is an instance of a 'normative variant' of the fallacy of affirming the consequent. The fallacy of affirming the consequent has a deductively invalid argument form of argument: if $A$ then $B ; B$; therefore $A$. An example would be: if I have graduated, I have paid my tuition fee; I have paid my tuition fee; therefore I have graduated. This form of reasoning is invalid form a viewpoint of deductive logic.

The advertising campaign called 'In Soviet Georgia', designed by the Burson ad agency, was run in various media. From 1975 through to 1978, these commercials were broadcast on American television, and print ads were run in magazines like Time and Newsweek. The commercial, called Son of Russia, written by Steve Kasloff, won the Clio award in 1978. The commercials presented shots of elderly Georgian farmers and the announcer said, 'In Soviet Georgia, where they eat a lot of yogurt, a lot of people live past 100'. Advertising Age ranked In Soviet Georgia as number 89 on its list of the best of 100 greatest advertising campaigns.

The text of the example to be analysed is the statement 'In Soviet Georgia, where they eat a lot of yogurt, a lot of people live past 100'. Two premises are explicitly expressed.

Explicit premise: In Soviet Georgia, they eat a lot of yogurt.

Explicit premise: In Soviet Georgia, a lot of people live past 100.

Similarly to the previous example, it would seem that in this case the conclusion is a prudential statement, 'You should eat yogurt'. However, the chain of reasoning in this case is a little more complex. We can analyse it by inserting some other implicit premises, and a secondary conclusion that links these premises to the ultimate conclusion.

Implicit premise: The eating of yogurt is causing the people in Soviet Georgia to live past 100.

Implicit conclusion: If you want to live longer, you should eat yogurt.

Implicit premise: You want to live longer.

Implicit conclusion: You should eat yogurt.

We can put all these elements together into an analysis by applying the argumentation scheme for argument from correlation to cause (Walton et al. 2008, p. 328).

Premise: $\quad$ There is a positive correlation between $A$ and $B$.

Conclusion: Therefore $A$ causes $B$.

The following are three critical questions for argument from correlation to cause.

$\mathrm{CQ}_{1}$ : Is there really a correlation between $A$ and $B$ ?

$\mathrm{CQ}_{2}$ : Is there any reason to think that the correlation is any more than a coincidence?

$\mathrm{CQ}_{3}$ : Could there be some third factor $C$, that is causing both $A$ and $B$ ?

This scheme is shown in the screen shot of the argument scheme selection menu of Araucaria in Figure 2. How the scheme fits the example is shown on the right. 


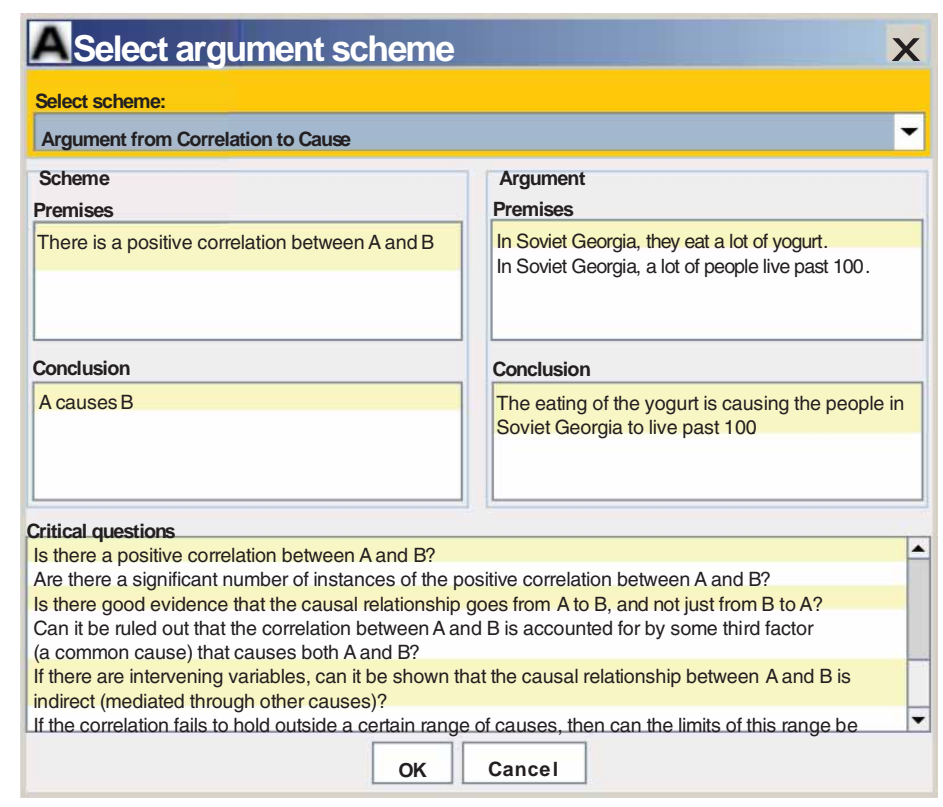

Figure 2. Screenshot of the argument scheme selection menu of Araucaria.

At the bottom of the screen shot of the menu in Figure 2 some of the critical questions matching the scheme for argument from correlation to cause are shown.

Now we can see how to analyse the argumentation in this case. The two explicit premises 'In Soviet Georgia they eat a lot of yogurt' and 'In Soviet Georgia a lot of people live past 100'. go together to support the implicit conclusion that the eating of the yogurt is causing the people in Soviet Georgia to live past 100. The argumentation scheme that binds these two premises together in support of the conclusion is argument from correlation to cause. But we can analyse the argument still further by showing how this argument leads to the conclusion that if you want to live longer you should eat yogurt. This conclusion, in turn, taken together with the implicit premise that you want to live longer, which can be seen as a goal premise, leads to the ultimate conclusion that you should eat yogurt. These two premises work together, based on the argumentation scheme for practical reasoning, to support the ultimate conclusion. The structure of the argumentation as a whole is displayed in Figure 3.

The analysis of this case is interesting because it shows not only an ad with an implicit conclusion, but one with an implicit sub-conclusion used to link one part of the argument with another. Also, two argumentation schemes can be applied to the structure of the chain of argumentation. We essentially have to chain two arguments connected to each other because an implicit conclusion of the one argument functions as a premise supporting the one premise in the other argument.

An interesting discussion point in the analysis of this particular example is whether the argument commits the post hoc fallacy, the error of leaping from a correlation to a premature causal conclusion. There are good grounds for concluding, on the analysis above, that the argumentation in this case does commit the post hoc fallacy. The analysis of it shown in the diagram in Figure, 3 , along with the argumentation scheme and list of critical questions given in Figure 2, provide the right kind of evidence needed to support such a criticism. So here we have a widely successful ad that, arguably, is an instance of the post hoc fallacy. But to go into the matter a little more deeply, we have to look at the context of dialogue. 


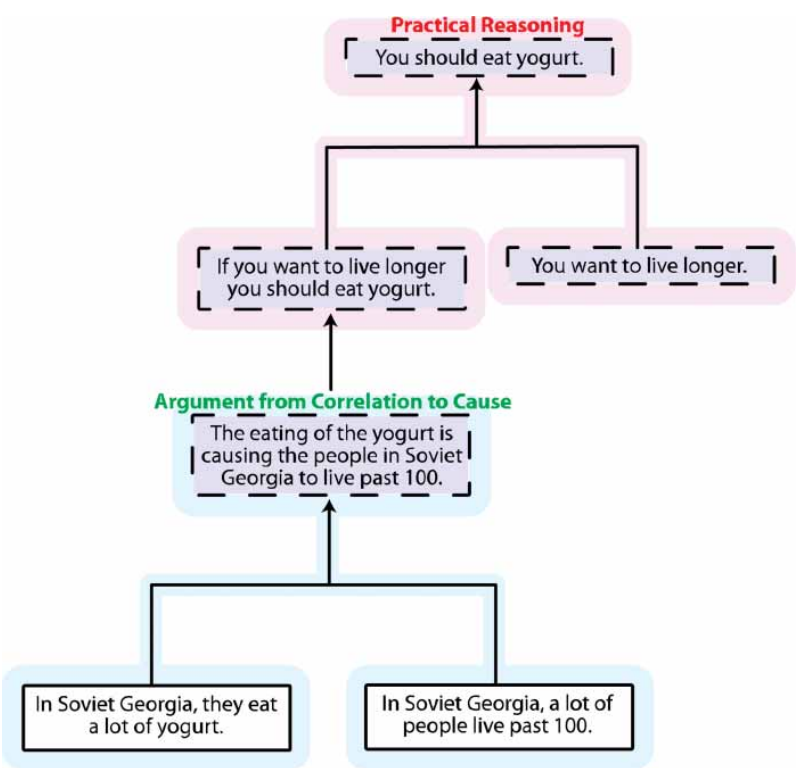

Figure 3. Araucaria visualisation of the argument in the Dannon yogurt example.

This ad was successful during an earlier time when people were aware of the longevity of the farmers in Georgia when it was widely thought to be a remarkable phenomenon because there appeared to be no explanation of why they would live so long. The ad exploited this common knowledge very successfully by allowing the reader to jump (precariously) to an explanation that served the marketers of yogurt products. The same ad would be very likely be laughable today, or at any rate would not be effective, as commonly held opinions about aging and nutrition have changed. As times have changed, so has the context in which ads are used for mass persuasion. Now there is an aging population that spends a lot on health-related products but also tends to be more sophisticated about health issues. For these readers, ads that are targeted to real health problems that they have, and address the problem by offering solutions that seem likely to work, based on practical reasoning of a kind that is not so obviously fallacious, may seem like a more effective persuasion strategy. We now turn to analysing a series of recent ads that seem to fit this trend.

Whether any of these is really fallacious or not is tricky, however, and depends on a number of general assumptions discussed in the concluding section. In the next two sections, several recent ads from Newsweek are analysed and discussed.

\section{The Lunesta, Mucinex and ACTOS examples}

There was no particular reason for selecting issues of Newsweek during the period of October 2007 to December 2009 as the main source of examples except that the author, a regular reader of Newsweek, had been struck by the number of ads appearing during this period that exhibited one particular type of argumentation, namely practical reasoning. Each ad in the series of examples in Sections 4 and 5 is meant to be a persuasive argument to get consumers to buy a product. This part of the project studies how each argument used particular schemes for this purpose, to evaluate strengths and weaknesses in the arguments by using the set of critical questions matching each scheme. The aims were to identify the argumentation strategies used in the ads so we can get a better idea of how they work as sequences of logical reasoning that can be broken down into parts 
and combined in argumentation structures. Each argument was visualised using an argument map that shows its parts, including its unstated premises or conclusions. This process helps us to see how the argumentation in the ads works.

This example is part of a lengthier chain of argumentation in an ad for the Lunesta sleep medication that appeared on the back cover of Newsweek, 8 October 2007. The picture in the ad showed the head and shoulders of a young man asleep, his head resting against the pillow. On his shoulder, a fluorescent butterfly was depicted. In large print, above the picture, the words 'The sleep you've been dreaming of' were printed. Below the picture, in smaller print but in capital letters, the expression 'Soothing Rest for Mind and Body' appeared. Just below that, the message containing the main argument of the ad appears. This text comprises most of the argument, but two sentences just after the part quoted have been deleted: 'It's what you've been craving. Peaceful sleep without a struggle. That's what Lunesta is all about: helping most people fall asleep quickly, and stay asleep all through the night'. It is easy to see that this text presents an argument directed towards getting the readers of the ad to buy Lunesta. However, it may be a little harder at first to see what are the premises that are put forward to support this conclusion, and what the form of the argument is. The argument evidently has some sort of structure, but it may not be apparent what that structure is. We begin by making a so-called key list of the statements that make up the explicit premises and conclusion of the argument.

Premise: Your goal is to have peaceful sleep without a struggle.

Premise: Taking Lunesta is the best means to have peaceful sleep without a struggle.

Premise: Lunesta helps most people fall asleep quickly.

Premise: They stay asleep all through the night.

When stated in this way, the argument can be analysed as having the form of the argumentation scheme called practical reasoning. This scheme represents goal-directed reasoning of the following sort: I have a goal; this action is a means to help fulfil the goal; therefore I should carry out this action. There are three basic components of this scheme. One premise describes an agent's goal. A second premise describes an action that the agent could carry out and that would be a means to accomplish the goal. The third component is the conclusion of the inference telling us that the agent should carry out this action.

Now we can analyse the argument in the Lunesta example by applying the scheme for practical reasoning to the statements in the key list. However, the main problem is that the conclusion does not appear to be stated explicitly in the given text. However, since the argument is part of an ad, we can reasonable take it that the purpose of the ad is to persuade the readership that taking Lunesta would be a good thing (from a prudential viewpoint) for them to do. The purpose of the ad is to sell product, and it looks like the argument is directed to this sort of conclusion. Hence in the analysis shown in Figure 4, we have inserted the conclusion as the implicit statement 'You should take Lunesta'.

In Figure 4, we can see how the two explicit premises are linked together, based on the scheme for practical reasoning, and work together to support the conclusion. The conclusion is displayed in a text box with a dashed border, indicating that the statement in the box is implicit. The remaining two statements, at the bottom of Figure 4, are depicted as providing two individual reasons, each of which stands on its own to support the statement above it, 'Taking Lunesta is the best means to have peaceful sleep without a struggle'. It is interesting to note that the implicit statement in this case is the conclusion, as contrasted with the more usual sort of case in which the implicit statement is one of the premises.

The Lunesta example is relatively simple, and represents a common kind of argument structure found in commercial ads for drugs, herbal products or foods that claim to have health benefits, 


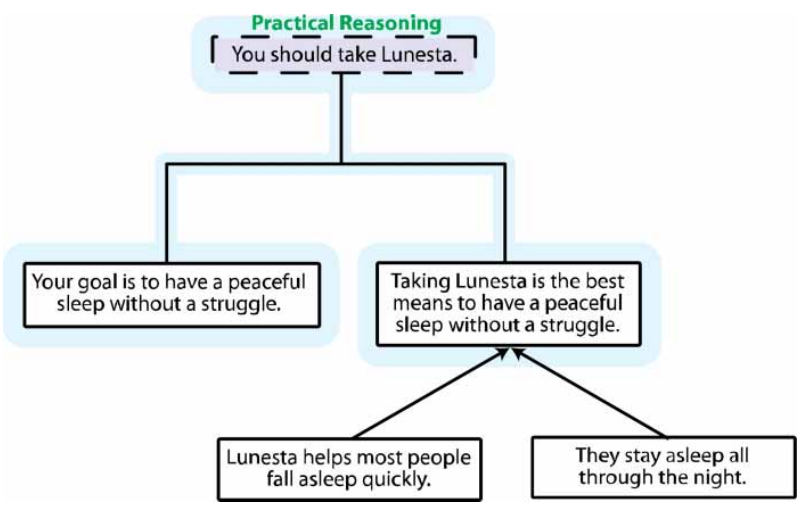

Figure 4. Araucaria visualisation of the argument in the Lunesta example.

except that it may be one of the premises in the argument rather than the conclusion that is implicit. We now turn to a brief mention of some other examples that are slightly more complex, and that raise interesting issues.

An ad for Mucinex shows a large character fashioned from what appears to be mucus (Newsweek, February 18, 2008, p. 5). The text under the visual reads, 'When mucus gives you major congestion, you need a major mucus fighter, new maximum strength Mucinex. Just one pill has the most mucus fighting medicine available, to break up and loosen congestion for a full $12 \mathrm{~h}$. In fact, it is the longest lasting non-prescription chest congestion medication you can buy. So when maximum mucus happens to you, overpower it with maximum strength Mucinex'. The basic argument in this ad can be put in the form of practical reasoning as follows: your goal is to reduce congestion by reducing the amount of mucus in your chest; taking maximum strength Mucinex is a means to realise this goal; therefore you should take maximum strength Mucinex. Another interesting aspect of the argumentation in the ad is that it mentions the claim that the product breaks up and loosens congestion for a full twelve hours. Then it states that the product is the longest lasting non-prescription chest congestion medication you can buy. This claim answers one of the critical questions matching the scheme for practical reasoning, namely the question of what alternative actions to the one being considered would also bring about the goal. It would very likely be known to both the reader of the ad and those who crafted it that there are competing products available that claim to achieve the same goal. So the consumer who reads the ad has a choice between different means of carrying out the goal, buying this product, or buying some competing product. This ad displays the interesting strategy of proleptic argumentation, the technique of putting forward an argument containing a reply to an objection even before the objection is made by the respondent is the argument. Proleptic argument amounts to making two moves at one turn in a sequence of dialogue. In this instance, the argument based on practical reasoning is put forward in such a way that it contains a reply to one of the critical questions matching the scheme.

It was stated above in the ACTOS example that critical questioning cannot be represented on the diagram, but there is a qualification to be made on this claim. It can be represented to some extent through the device of refutation. Let us return to the Mucinex example to show how, using Figure 5. The basic Mucinex argument is shown on the left as an instance of practical reasoning. The statement in the darkened box with the dotted border is shown joined to the practical reasoning argument by a double arrow. The double arrow stands for what is called refutation in Araucaria, which is supposed to be like negation. The statement in this text box, stating that there might be a better product for this purpose than Mucinex, operates like the asking of a critical question matching the practical reasoning argumentation scheme, namely the critical question of what alternative actions to the one being considered would also bring about the goal. 


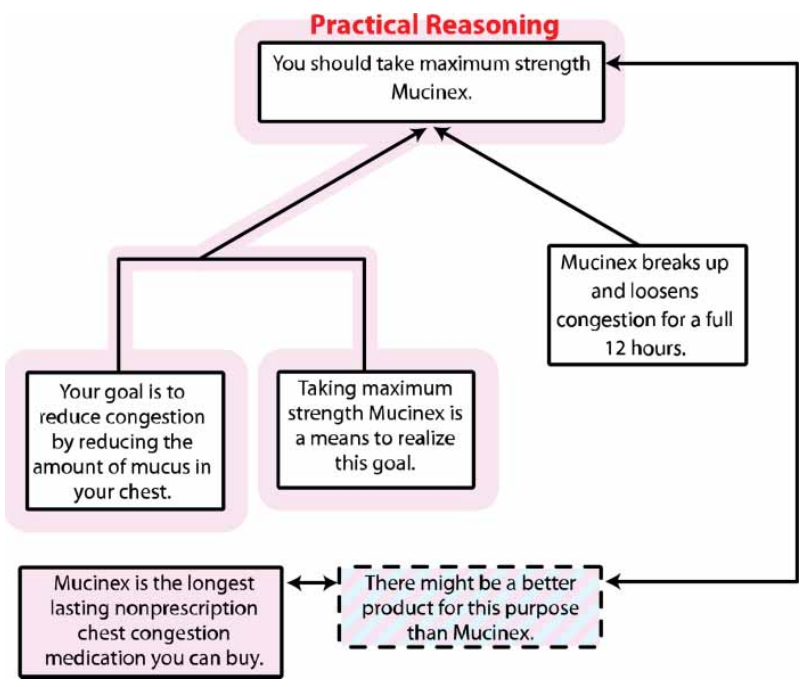

Figure 5. Using refutation to represent answering a critical question.

Hence the device of refutation does allow us to express the notion of a critical question being asked in response to an argument matching a particular scheme. However, Araucaria treats the refutation as being a statement, and no distinction is drawn between making a statement and asking the question. Then the statement which appears in the left most darkened box, stating that Mucinex is the longest lasting non-prescription chest congestion medication you can buy, is drawn as a refutation of the refutation that appears to its right. This example illustrates the refutation of a refutation, in other words.

This ad has an interesting visual aspect. The mucus man is a burly and threatening looking figure who also looks mildly disgusting. He is portrayed as somebody or something bad, who is about to do harm to you (the reader of the ad). This visual part of the ad uses value-based practical reasoning. The argument is telling the viewer that he needs to take steps to protect himself from this bad individual, and the way to do that is to buy Mucinex. The visual part of the ad highlights the written text in the ad by drawing the reader's attention to a bad problem he might have, and to a way of solving it.

An ad for ACTOS, a medication for diabetes (Newsweek, November 26, 2007, p. 25), has the headline: 'ACTOS has been shown to lower blood sugar without increasing the risk of having a heart attack or stroke'. The ad presents ACTOS as a way for the reader who has type 2 diabetes to solve the problem of lowering his/her blood sugar. It expresses this sort of argument: 'you have the goal of lowering your blood sugar; taking ACTOS is a means to realise this goal; therefore you should take ACTOS'. The ad also responds to critical questions proleptically (in advance of their being put forward), by including a response to $\mathrm{CQ}_{5}$ to the effect that the negative consequences of increasing the risk of heart attack or stroke will not occur.

Critical questioning cannot (straightforwardly) be represented on the diagram, but the potential rebuttal could be diagrammed as a pair of arguments fitting the scheme for argument from negative consequences mentioned in Section 1. An argument diagram showing how the proleptic argumentation in the ACTOS example works is presented in Figure 6.

The basic practical reasoning structure of the argument is shown on the bottom, displaying the argumentation scheme for practical reasoning as applied to the argument. The statement 'Therefore you should not take ACTOS' is displayed at the top in a darkened box, indicating refutation in Araucaria. Refutation is something like negation, indicated in Araucaria by a double headed 


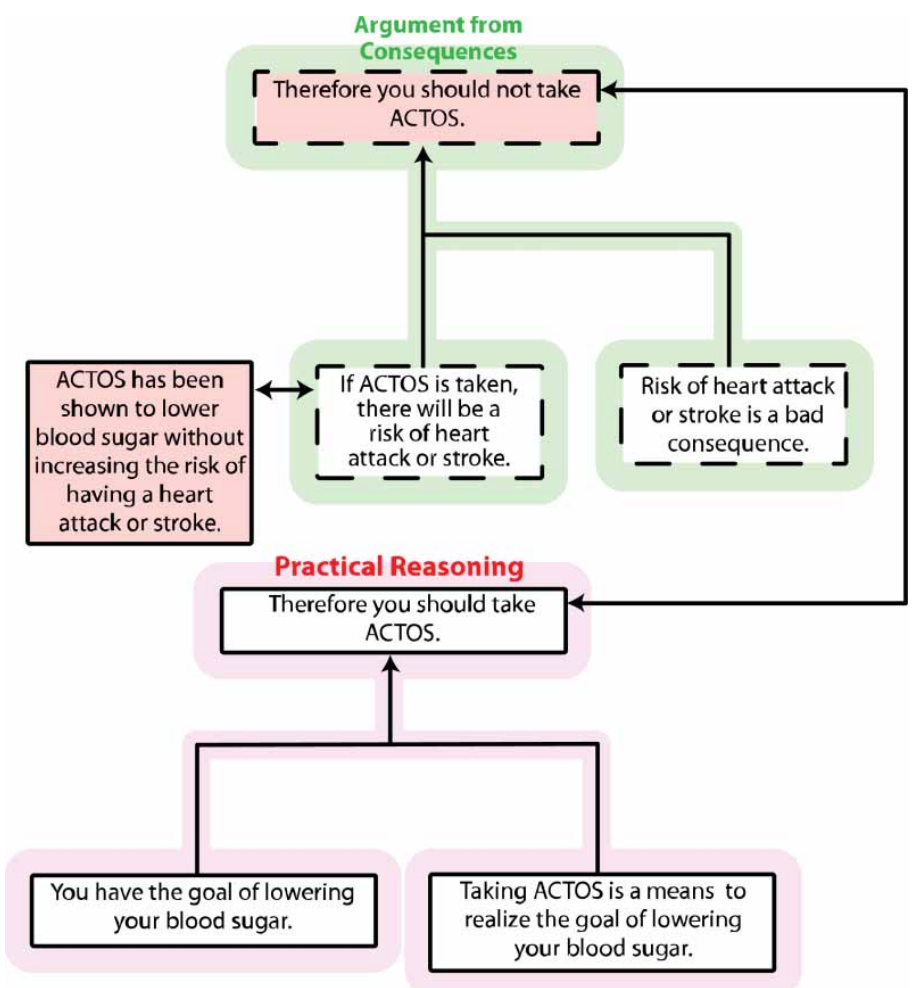

Figure 6. Argument diagram for the ACTOS example.

arrow. The refutation is supported by argument from consequences, as shown in the middle. What is also shown is that the premise 'If ACTOS is taken there will be a risk of heart attack or stroke' is itself refuted by another claim. This is shown by the statement in the darkened box at the middle left of Figure 6.

\section{The Caduet, PLAVIX and Lap-Band examples}

Argumentation from negative consequences is extremely common in the Newsweek ads where it is used to cite possible side effects of a medication, or as in the ACTOS example, to argue proleptically. Some of the ads deal at great length with possible side effects of taking the medication advertised. For example, an ad for Caduet (Newsweek, December 29, 2008, p. 29), a drug promoted as one pill that reduces both high blood pressure and high cholesterol, offers many details concerning side effects. In a section entitled Possible Side Effects of Caduet, it lists headache, constipation, swelling of the legs or ankles, gas, feeling dizzy, and upset stomach and stomach pain. It also mentions unexplained muscle weakness, nausea, vomiting, brown or dark coloured urine, feeling more tired than usual, and the skin and whites of your eyes turning yellow.

The next ad shows a picture of a woman, and beneath that it says, 'I have poor leg circulation. And I have a good reason to try to reduce the risk of heart attack or stroke that comes with it'. Further below, more argumentation is presented.

Peripheral artery disease (PAD) is often described as poor leg circulation, which puts you at the double risk of heart attack or stroke. That is because, if you have poor blood circulation in your legs, you may 


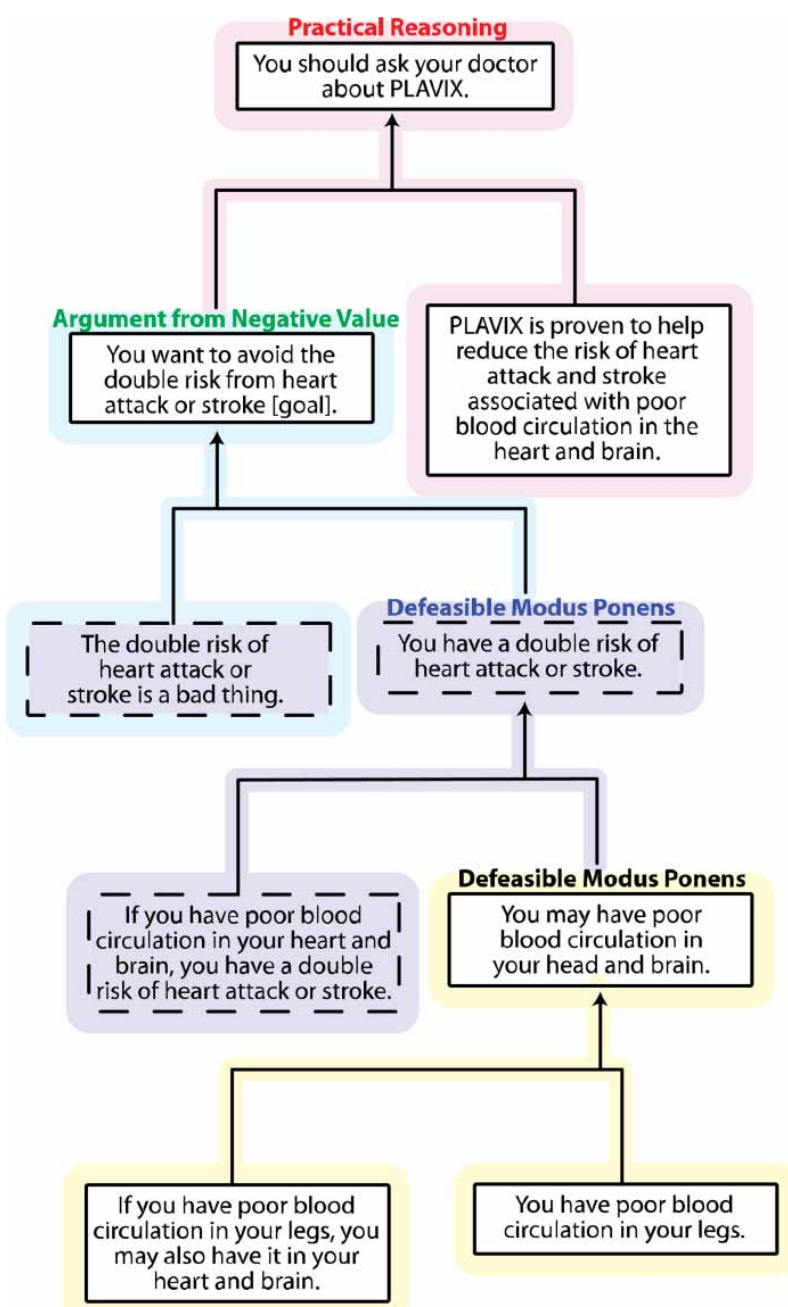

Figure 7. Argument diagram of the PLAVIX example.

also have it in your heart and brain. You may feel nothing, but the most common system symptom of PAD is pain or heaviness in the legs. Take the next step. So if you are diagnosed with PAD, ask your doctor about a treatment clinically proven to help reduce your risk of heart attack and stroke associated with PAD. PLAVIX helps keep blood platelets from sticking together and forming dangerous clots, the cause of most heart attacks and strokes. Ask your doctor about PLAVIX.

This chain of argumentation is fairly complex, and there could be many ways to diagram it, but one simple way that captures the practical reasoning structure is shown in Figure 7.

The three statements in the darkened boxes with the dashed lines around them are implicit premises that have been inserted. The explicit premises are listed as follows.

You want to avoid the double risk of heart attack or stroke [goal].

If you may have poor blood circulation in your legs, you may also have it in your heart and brain. You have poor blood circulation in your legs.

You may have poor blood circulation in your heart and brain. 


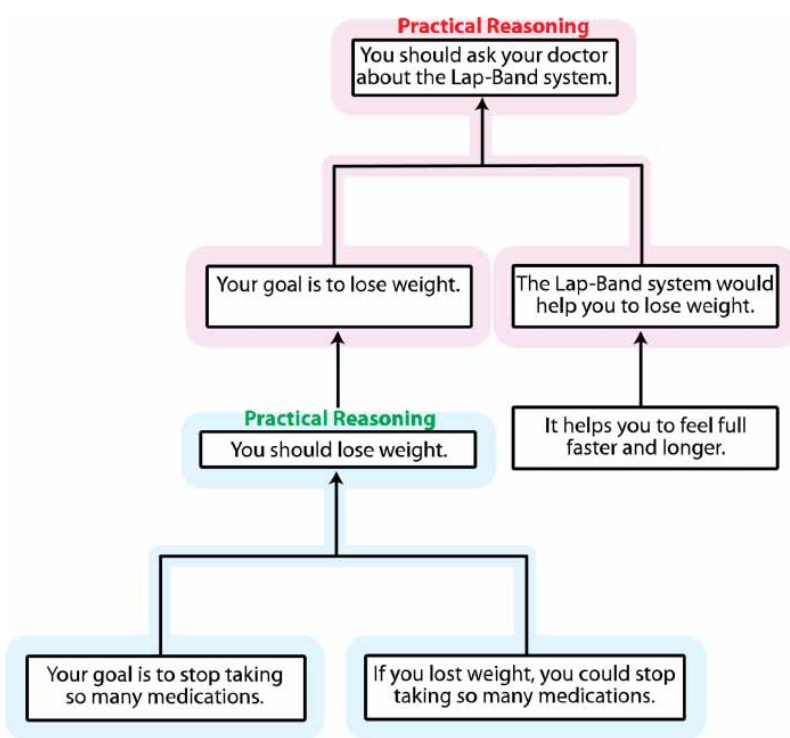

Figure 8 . Visualisation of the practical reasoning in the Lap-Band example.

PLAVIX is proven to help reduce the risk of heart attack and stroke associated with poor blood circulation in the heart and brain.

You should ask your doctor about PLAVIX.

In one instance, an implicit premise also plays the role of an implicit conclusion by forming a chain of reasoning. This form of argument is illustrated by the goal premise in the practical inference at the top of Figure 7, supported by argument from values.

The argument in the next ad is an interesting contrast with the one in the Dannon yogurt example. This ad is a more recent one (Newsweek, September 28, 2009, p. 61) and has several interesting features that are also different from any of the other ads studied above. It shows a large picture of an attractive young woman who appears to be visibly overweight. The text of the ad is printed against the backdrop of the picture of the young woman. Two sentences are displayed in large text in the middle of the page. One says: 'If I lost the weight, I could stop taking so many medications'. The other says: 'So I talked to my doctor about the Lap-Band system'. The next part of the text is quoted below.

If you're ready to finally lose weight and keep it off, then you may be ready for the Lap-Band System. The Lap-Band system is a device that's placed around the upper part of the stomach - often as an outpatient procedure - to help you feel fuller faster and longer. It's a healthy way to lose a significant amount of weight, and enjoy long-term results! Unlike gastric bypass surgery, there's no stomach cutting or stapling, plus it's adjustable for your needs, and can even be removed if necessary.

The remainder of the ad is concerned with contraindications and possible side effects. For example, it is stated that the system is not for those who are pregnant or suffer from certain disorders, and that band slippage, stomach injury, vomiting and heartburn may occur. On an adjoining page (p. 60) a detailed list of contraindications, warnings and possible adverse effects is presented.

One of the interesting aspects of this ad is that it presents the young woman whose picture is displayed in the ad as an example of someone who has taken this medical treatment, and is offering advice to the reader of the ad, supposedly based on her personal experience. It is interesting because the woman pictured in the ad has certain goals that are either stated or may be attributed to her, 
and the assumption is that certain target readers of the ad to whom its argumentation is directed are presumed to share these goals. The woman pictured in the ad has supposedly carried out an action, namely taking this treatment, and it has enabled her to successfully achieve these goals. One of her goals, prominently stated in the large print at the beginning of the ad, was for her to stop taking so many medications. It is presumed that a significant number of potential readers of the ad would be in the same position as this young woman, namely that of taking a lot of medications that they would like to stop taking.

Thus the ad has a dialogue structure with two levels of practical reasoning in it. One is the young woman's description of her own process of practical reasoning, whereby she took steps to go ahead with this treatment based on her goal of stopping taking so many medications. The assumptions are that she is in a position to know about the treatment because she has actually taken it, and that the target readers of the ad who are in the same position can benefit from her experience. There are also some implicit goals that can be seen as enthymematic premises. Although it is not stated explicitly in the wording of the ad, it is clear that her goal was to lose weight. There is also an implicit assumption that taking so many medications is undesirable. It is well known that taking medications has side effects that can be made more complicated, and even dangerous, if the side effects conflict with each other in ways that may be impossible to predict. This can be presumed to be common knowledge among the readers to whom the ad was directed.

In the main argument, quoted above, several subarguments may be distinguished. One is that the device is installed as an outpatient procedure, as opposed to gastric surgery that has to take place in a hospital, a procedure that presumably most people would be less comfortable with. Another is the statement that it helps the person feel full faster and longer, a premise that shows how the technique works, and makes the claim that it does work. Finally, other advantages over gastric bypass surgery are cited: no stomach cutting or stapling, it's adjustable for your needs, and it can be removed if necessary. The conclusion of the ad directed to the reader is that if he or she is in the same position as the young woman pictured in the ad, and has the same goals, he or she should consider this treatment. More specifically, the reader of the ad is advised to take the first step of talking to his or her doctor about taking this treatment.

The practical reasoning part of the argument can be diagrammed as shown in Figure 8. This part of the argumentation is interesting because it is an example of chaining of practical inferences into a sequence of practical reasoning.

Some other parts of the argumentation are also interesting to comment on. The critical question of whether there are better alternatives to the proposed course of action is replied to by several arguments. One alternative that is mentioned is gastric bypass surgery. The ad states that unlike gastric bypass surgery, there is no stomach cutting or stapling. The implicit value premise is that stomach cutting or stapling is a negative value for the reader of the ad. These would be taken to be negative consequences of any weight loss treatment being considered. Hence they are reasons for the Lap-Band system and against the alternative of gastric bypass surgery. Two other reasons are given to support the Lap-Band system against other possible alternatives. It is adjustable for your needs, and it can even be removed if necessary.

The general contrast between this example and the yogurt example is interesting to observe. The latter example could be used to illustrate deceptive argumentation uses in an ad. The argument even arguably commits a fallacy. The argumentation used in the Lap-Band ad seems highly reasonable. It makes a number of claims, but they seem plausible enough and are supported by evidence that is given. It offers a considerable body of factual information concerning contraindications and possible side effects of the treatment. As shown in Figure 8, the ad is based on two instances of practical reasoning that chain forward to the ultimate conclusion in the example that I should ask my doctor about the Lap-Band system. On the whole, one could say, the argumentation in the sequence of practical reasoning seems quite reasonable. Not only is it based on argumentation 
schemes, but it goes to considerable lengths to answer relevant critical questions that match the schemes.

\section{The charge that ads widely tend to commit fallacies}

There is a commonly held general assumption in logic that commercial ads of the kind studied tend to be based on questionable emotional appeals and fallacious arguments, as contrasted with genuine logical reasoning that contains factual information of the kind useful to a consumer considering taking steps to solve a practical problem (Slade 2003). Many examples of commercial ads are commonly cited in logic textbooks in the sections on informal fallacies as examples of fallacious arguments. As shown in Section 2, it has been argued that these ads commit fallacies.

Birdsell and Groarke (1996) and Slade (2003) were quite right to draw attention to the significance of the visual aspect of argumentation in ads. Images were used in all the ads studied in Sections 4 and 5, although in some instances they were highly significant and in other cases minimal. Some were highly evocative emotionally and were directed towards values of the targeted readers. The Lunesta butterfly hovering over the sleeper's pillow evokes drug safety. The Mucinex man evokes mild disgust towards mucus. The Mucinex ads varied over the period studied with different depictions of the mucus man as a hooligan attacking the cold sufferer but being repelled by Mucinex. In one such ad (Newsweek, December 7, 2009, p. 35), the mucus man is depicted as a muscular, threatening biker being blown off his motorcycle by Mucinex. Here some mild emotions of fear are appealed to, but in the Plavix ad, the fear of clogged arteries is graphically exploited by a picture of a blood vessel being plugged up by cholesterol. The reader can easily imagine the consequences of this situation. These pictures in the ad can be analysed as putting arguments forward (Slade 2003; Groarke 2009), but they also perform a function of drawing the reader's attention to a problem that may affect him and that needs to be solved. Presumably, most readers of Newsweek ignore the ads, or pay little attention to them, but a graphic that relates to their specific problems will grab attention.

The visual aspect of argumentation in the ads is one focus of the allegation that the ads commit logical fallacies. Groarke's example of the giant vodka bottle being poured onto the sleepy village, described in Section 3, was taken to express the following argument: [Explicit Premise] If you add vodka to your life, your sleepy life will be transformed into a life of cosmopolitan excitement; [Implicit Premise] A life of cosmopolitan excitement is desirable; [Conclusion] You should add vodka to your life (i.e. purchase vodka). Groarke evaluated this argument as fallacious by classifying it as an instance of the fallacy of affirming the consequent. It is questionable however whether this is the best way of representing the form of this argument, for the implicit premise is about whether a life of cosmopolitan excitement is desirable. A better way of representing the form of the argument is as an instance of practical reasoning. The goal is a life of cosmopolitan excitement, the means is to employ vodka, and the conclusion is to buy vodka. Instead of measuring the argument against the standard of deductive validity, we can view it as fitting the argumentation scheme for practical reasoning.

Seen as an instance of defeasible practical reasoning, the argument is not fallacious, or at any rate the basic structure of the argumentation in the ad is reasonable. As an instance of practical reasoning, it can be seen as reasonable depending on two assumptions: (1) whether the premises are true and (2) whether it takes the appropriate critical questions for practical reasoning into account. It can be argued that it fails on both counts, and indeed, Groarke's criticisms of some of the weak points in the argument can be nicely fitted into this format. For example, it can be pointed out that there are negative consequences of drinking vodka, it can be argued that the excitement it provides is temporary, and so forth. Perhaps there are grounds for alleging that the example is 
an instance of fallacious use of practical reasoning, as well, but by categorising the argument as a deductive one that needs to meet standards for deductive reasoning, Groarke's analysis fails to recognise that the underlying structure of the argument is that of practical reasoning, a defeasible form of argument that is most fully evaluated by seeing how well it stands up to critical questioning based on its argumentation scheme.

As shown at the end of Section 2, pharmaceutical health product ads have come under even more detailed criticism for committing fallacies. Using methods of argumentation theory to analyse examples of ads for pharmaceutical products, Rubinelli (2005) concluded that some of the arguments in the ads can be said to have committed informal fallacies. She cited an ad for Allegra that failed to include information about side effects of taking the product, possibly leading someone to ask his/her doctor for a medication that is not appropriate for them: 'We are here dealing with a clear fallacy of omission, based on a failure to present information' that is relevant for consumers (Rubinelli 2005, p. 89). Rubinelli et al. (2007, p. 1213) cited an ad that claimed that the product advertised is better than the comparable products on the market, but provided no support for this claim. They also cited ads (p. 1213) that either failed to mention side effects, or printed them in a small font using technical language that would not be familiar to all readers. The fallacy is taken to be a failure to provide relevant information. One general failure of the DTCA ads they cited is that they commit the fallacy of omission, based on their failure to present information to consumers (Rubinelli et al. 2007, p. 1214). A further series of DTCA ads analysed in Rubinelli et al. (2008, p. 53) were said to show that the ads commit a fallacy of relevance by emphasising certain aspects in a more inviting way, for example by using larger print in a more prominent place, 'inviting the reader to ignore crucial information'.

The underlying issue is whether a failure to present information in an argument in an ad is fallacious. The answer depends on whether the purpose of the ad, or part of it, is to present information to the reader. Supporters of DTCA see it as a way of providing people with information about the benefits and risks of medication, arguing that pharmaceutical companies have more scientifically-based information than any other sources (Rubinelli et al. 2008, p. 49). However, detractors emphasise the financial interests of the pharmaceutical companies, arguing that their aim is to sell product by creating brand recognition (Rubinelli 2005, pp. 76-77). Hence a good part of the dispute seems to be about the supposed purposes of this type of advertising. Hence to properly judge whether arguments used in such health product ads are fallacious or not, we have to look at the context of use of the ad in a type of dialogue.

Omitting to present information in an argument is not always fallacious. It depends on whether the unstated information is relevant, and this in turn depends on what type of dialogue the argument is supposed to be part of. Proof standards are different in different types of dialogue (Gordon and Walton 2009). The proof standard in a scientific investigation, or in evidence-based medicine, should properly be higher than the proof standard required for an argument placed in an ad in magazine. Given this difference, it was remarkable that the Newsweek ads presented so much information on side effects. Even if such an ad fails to tell the reader about all the possible side effects of taking the medication in question, inferring that the argument used in the ad therefore commits a fallacy of omission is a logical leap. Sometimes omitting information can be reasonable in argumentation, depending on what the goal of the dialogue is supposed to be.

\section{Conclusions}

It is true that pictures (graphics) can appeal to emotion more directly and more powerfully that words, especially if combined with words, and that pictures contain arguments in many instances (Birdsell and Groarke 1996). But should appeals to emotion of these kinds be treated as fallacious arguments just because they appeal to emotion? Should emotional thinking always be treated as 
the opposite of logical reasoning? Is an appeal to emotion always logically defective as a good reason for accepting a conclusion? For a long time, the answer to these questions was affirmative, but more recently it has come to be recognised that the traditional blanket condemnation of appeals to emotion in argumentation is not warranted (Walton et al. 2008). Appeals to emotion should be generally recognised as having legitimate standing as being, under the right conditions, reasonable arguments carrying some weight in shifting a burden of proof in a balance of considerations case where exact calculation of the outcome is not a practical possibility. It is necessary to judge the argument in each ad by evaluating the purpose of its use in a dialogue context.

Consumers in a free market economy are not so naïve that they do not know that the ads are using all kinds of clever strategies to try to get them to pay attention to the message in the $\mathrm{ad}$, to remember it, and to view the product favourably. Both sides in the persuasion dialogue know the game. Practical reasoning is a reasonable form of argumentation if used properly. The examples from Newsweek analysed in Sections 4 and 5 suggest that the underlying argumentation for practical reasoning used in the problem/solution ads is inherently reasonable, even though the standard of rationality appropriate for its use is not the same as the standards used in traditional deductive and inductive reasoning. Certainly it is the dominant argumentation scheme used in the ads, and by far the most common scheme that was identified. The examples studied also show how the scheme for practical reasoning is used in these health ads in interesting ways by combining it with other schemes and with the use of enthymemes. The examples show how schemes can help reveal implicit premises and conclusions in the arguments.

The yogurt example shows how an analysis can help to uncover implicit assumptions in a chain of reasoning that should be open to critical questioning. The fourth critical question, which asks if the correction could be accounted for by some other factor than the one cited as cause, is especially important in this case. In this instance, it would be fallacious to jump to the conclusion that the eating the yogurt is the cause of the longevity. Drawing this conclusion is questionable, given that many other factors, like environment and life style, not to mention other foods, need to be taken into account. Hence the argument in this example can justifiably be judged to be fallacious.

The arguments in the ads studied in Sections 4 and 5, in contrast, are basically reasonable, in virtue of their using practical reasoning to offer the reader of the ad a solution to a problem that he or she might have. What is shown is that there is a current trend to base the argumentation on practical reasoning, and that this kind of reasoning is not fallacious in the way claimed, even though it may be based on premises that are not always explicit, or not very well substantiated. What has been shown is a way to help overcome deficiencies in techniques of tailored health communication in consumer health informatics by using argumentation schemes, argument visualisation tools, and dialogue models to frame these persuasive communication messages. The evidence collected has been shown to be useful to help explore the planning mechanisms that account for the generation of text consisting of multiple arguments in health product communications.

\section{References}

Atkinson, K., Bench-Capon, T., and McBurney, P. (2006), 'Computational Representation of Practical Argument', Synthese, 152, 157-206.

Bench-Capon, T. (2003), 'Persuasion in Practical Argument Using Value-based Argumentation Frameworks', Journal of Logic and Computation, 13, 429-448.

Bench-Capon, T., and Atkinson, K. (2009), 'Abstract Argumentation and Values', in Argumentation and Artificial Intelligence, eds. I. Rahwan and G. Simari, Berlin: Springer, pp. 45-64.

Birdsell, D.S., and Groarke, L. (1996), 'Toward a Theory of Visual Argument', Argumentation and Advocacy, $33,1-10$.

Bratman, M., Israel, D., and Pollack, M. (1988), 'Plans and Resource-Bounded Practical Reasoning', Computational Intelligence, 4(3), 349-355. 
Burke, M. (1985), 'Unstated Premises', Informal Logic, 7, 107-118.

Calfee, J.E. (2002), 'Public Policy Issues in Direct-to-Consumer Advertising of Prescription Drugs', Journal of Public Policy \& Marketing, 21(2), 174-193.

Copi, I., and Cohen, C. (2005), Introduction to Logic (12th ed.), Upper Saddle River: Pearson Educational.

Ennis, R. (1982), 'Identifying Implicit Assumptions', Synthese, 51, 61-86.

Gordon, T.F., and Walton, D. (2009), 'Proof Burdens and Standards', Argumentation and Artificial Intelligence, eds. I. Rahwan and G. Simari, Berlin: Springer, pp. 239-260.

Gough, J., and Tindale, C. (1985), 'Hidden or Missing Premises', Informal Logic, 7, 99-106.

Groarke, L. (2009), 'Informal Logic. Stanford Encyclopedia of Philosophy', ed. Edward N. Zalta. http://plato.stanford.edu/entries/logic-informal

Hamblin, C. (1970), Fallacies, London: Methuen.

Hitchcock, D. (1985), 'Enthymematic Arguments', Informal Logic, 7, 83-97.

Kukafka, R. (2005), 'Tailored Health Communication', in Consumer Health Informatics: Informing Consumers and Improving Health Care, eds. D. Lewis, G. Eysenbach, Z. Stavri, and H. Jimison, New York: Springer, pp. 22-33.

Lexchin, J., and Mintzes, B. (2002), 'Direct-to-Consumer Advertising of Prescription Drugs: The Evidence Says No', Journal of Public Policy \& Marketing, 21(2), 194-201.

Prakken, H. (2006), 'Formal Systems for Persuasion Dialogue', The Knowledge Engineering Review, 21, 163-188.

Reed, C., and Rowe, G. (2002), 'Araucaria: Software for Puzzles in Argument Diagramming and XML', Technical Report, Department of Applied Computing, University of Dundee. http://www.computing. dundee.ac.uk/staff/creed/araucarial

Reed. C., and Rowe, G. (2006), Araucaria, Version 3_1. http://www.computing.dundee.ac.uk/staff/creed/ araucarial

Rubinelli, S. (2005), 'Ask Your Doctor: Argumentation in Advertising of Prescription Medicines', Studies in Communication Sciences, 5(2), 75-98.

Rubinelli, S., Nakamoto, K., and Schulz, P.K. (2007), 'Reading Direct-to-Consumer Advertising of Prescription Medicine: A Qualitative Study from Argumentation Theory on its Dialectical and Rhetorical Features', in Proceedings of the Sixth Conference of the International Society for the Study of Argumentation, eds. Frans van Eemeren et al., Amsterdam: SicSat, pp. 1211-1215.

Rubinelli, S., Nakamoto, K., and Schulz, P.K. (2008), 'The Rabbit in the Hat: Dubious Argumentation and the Persuasive Effects of Prescription Drug Advertising', Communication \& Medicine, 5(1), 49-58.

Scheuer, O., Loll, F., Pinkwart, N., and McLaren, B.M. (2009), 'Computer-supported Argumentation: A Review of the State of the Art', International Journal of Computer-Supported Collaborative Learning, $5(1), 1-67$.

Scriven, M. (1976), Reasoning, New York: McGraw-Hill.

Slade, C. (2003), 'Seeing Reasons: Visual Argumentation in Advertisements', Argumentation, 17, 145-160.

Walton, D. (1996), Argumentation Schemes for Preusmptive Reasoning, Mahwah, NJ: Routledge.

Walton, D. (2007a), 'Evaluating Practical Reasoning', Synthese, 15, 197-240.

Walton, D. (2008), 'Three Bases for the Enthymeme: A Dialectical Theory', Journal of Applied Logic, 6, 361-379.

Walton, D., and Krabbe, E. (1995), Commitment in Dialogue, Albany: State University of New York Press.

Walton, D., and Reed, C. (2005), 'Argumentation Schemes and Enthymemes', Synthese, 145, 339-370.

Walton, D., Reed, C., and Macagno, F. (2008), Argumentation Schemes, Cambridge: Cambridge University Press.

Wooldridge, M. (2000), Reasoning about Rational Agents, Cambridge, MA: The MIT Press. 